\title{
Automatic system of diagnosing and treatment in intensive care unit
}

\author{
R Solodova ${ }^{1 *}$, M Sokolov' ${ }^{1}$ V Galatenko ${ }^{1}$, M Petrova ${ }^{2}$ \\ From ESICM LIVES 2015 \\ Berlin, Germany. 3-7 October 2015
}

\section{Introduction}

According to statistics, in average 1.7 medical errors occurs daily in the treatment of patients in intensive care [2]. 78\% of medical errors are drug dosing errors [3]. Such errors can occur at any stage of the treatment, whether use of the drug, its preparation or administration. Mistakes in medicinal treatment had been done in 187 cases out of 5744 (3.3\%) [1]. The greatest number of errors are related to speed of administration (40.1\%), a pass-dose, incorrect dosage and delayed use of the drug were, respectively, $14.4 \%, 11.7 \%$ and $13.9 \%$ [1]. The automatic robotic system is intended to reduce the number of medical errors by automatic dose calculation due to weight, age and etc. It consists of three functional modules: a block of drug administration, patient monitor, the analytical unit (decision support system - processing of received information, the definition of the state, providing recommendations). Data from all devices are automatically directed to the program and program sends commands to pumps. The system is based on national clinical guidelines of patient care in intensive care unit.

\section{Objectives}

The aim of the study was to evaluate the effectiveness of diagnosis and correct treatment sequence of the automatic system.

\section{Methods}

The system performance testing was carried out on patients in intensive care unit. All diagnostic probes (pulse, ECG, blood pressure) of the system was connected to 53 patients on admission. System asks questions step by step and analyze physiological data, make a diagnosis and suggest treatment, after confirmation by the doctor it starts infusions. System corrects velocity of drug administration according to the values of heart rate, saturation, blood pressure.

\section{Results}

In 38 cases system established diagnosis and it was admitted by the doctor, in 2 cases it was not confirmed by the doctor. In 13 cases, the diagnosis of the patient was beyond worked (thromboembolia of pulmonary artery, asthmatic status, cardiopulmonary edema, acute coronary syndrome, hypoglycemic coma, septic and hemorrhagic shock, arrhythmias). The treatment offered by the system was accepted in all diagnosed cases. There were none side effects or unwanted sequels.

\section{Conclusions}

Robotic system helps to diagnosis in $95 \%$ of cases and suggest about first therapeutic actions. In time sensitive cases it can prevent misdiagnosis and improper treatment. Further accumulation of material is need to prove reduction in number of medical errors.

\section{Grant Acknowledgment}

Research supported by contract No02.G25.31.0030.

\section{Authors' details}

${ }^{1}$ Lomonosov Moscow State University, Moscow, Russian Federation.

${ }^{2}$ People's Friendship University of Russia, Moscow, Russian Federation.

Published: 1 October 2015

References

1. Calabrese AD: Medication administration errors in adult patients in the ICU. Intensive Care Med 2001, 27:1592-8.

2. Donchin $Y$ : A look into the nature and causes of human errors in the intensive care unit. Crit Care Med. Crit Care Med 1995, 33:294-300.

3. Rothschild JM: The Critical Care Safety Study: the incidence and nature of adverse events and serious medical errors in intensive care. Crit Care Med 2005, 33:1694-700.

\section{doi:10.1186/2197-425X-3-S1-A729}

Cite this article as: Solodova et al: Automatic system of diagnosing and treatment in intensive care unit. Intensive Care Medicine Experimental 2015 3(Suppl 1):A729. 